\title{
Flurbereinigung: Privatnützigkeit und Ökosystemleistungen
}

\author{
Land consolidation: Private benefit and ecosystem services*
}

Stefan Möckel und André Wolf

(c) Der/die Autor(en) 2022. Dieser Artikel ist eine Open-Access-Publikation.

Flurbereinigung diente lange Zeit der Umgestaltung des ländlichen Raums hin zu intensiv genutzten Agrar- und Forstlandschaften. Sie ist aufgrund der rechtlichen Festsetzungsmöglichkeiten aber auch hervorragend geeignet, diese Landschaften nunmehr an den Klimawandel und die sonstigen ökologischen Nachhaltigkeitserfordernisse anzupassen, da sie u. a. die Wiederherstellung von Landschaftselementen und Habitaten für wildlebende Arten, die Schaffung guter Zustände bei Oberflächengewässern und Grundwasserständen sowie die eigentumsrechtliche Arrondierung von Schutzgebieten ermöglicht. Rechtlich müssen Flurbereinigungsverfahren allerdings vorrangig den Interessen der beteiligten Grundstückseigentümer dienen (Privatnützigkeit), sofern nicht die Voraussetzungen für eine enteignende Unternehmensflurbereinigung vorliegen. Maßnahmen des Umwelt- und Naturschutzes wurden bisher meist nicht als privatnützig angesehen, was den Anwendungsbereich des Instruments einschränkte. Mit der Anerkennung und Identifizierung von Ökosystemleistungen könnte sich jedoch der Anwendungsbereich deutlich erweitern, da viele dieser Leistungen auch oder sogar in erster Linie den Grundstückseigentümern zugutekommen. Der Aufsatz zeigt, in welchem Umfang die verschiedenen Verfahren des Flurbereinigungsrechts die Belange des Umwelt- und Naturschutzes mit aufgreifen und inwieweit Maßnahmen zur Wiederherstellung von Okosystemen und ihrer Leistungen privatnützige, gemeinschaftliche Anlagen darstellen.

\section{Einleitung}

Mehr als 50 Prozent der terrestrischen Ökosysteme in Deutschland unterliegen einer landwirtschaftlichen Nutzung und weitere 30 Prozent werden größtenteils forstwirtschaftlich genutzt ${ }^{1}$ Im europäischen Vergleich weist Deutschland hierbei einen sehr hohen Flächenanteil an Intensivlandwirtschaft auf. ${ }^{2}$ Intensiv bewirtschaftete Agrarlandschaften kennzeichnet, dass in den letzten hundert Jahren zugunsten höherer Erträge und einer maschinellen Bewirtschaftung insbesondere:

- Schläge vergrößert, Böden melioriert und Landschaftselemente (z.B. Hecken, Ufergehölze, Baumreihen und -inseln) entfernt wurden,

- mittels Entwässerungsgräben und Drainagen der Oberflächenabfluss erhöht und die Grundwasserspiegel abgesenkt sowie Moorböden kultiviert wurden und

- Fließgewässer begradigt, verlegt oder kanalisiert sowie Kleingewässer beseitigt wurden. ${ }^{3}$

Zusammen mit dem intensiven Einsatz von Dünge- und Pflanzenschutzmitteln zählen die landschaftlichen Umgestaltungen zu den wichtigsten Ursachen für den Rückgang wildlebender Arten (z.B. Insekten, Vögel, Pflanzen) in Agrarlandschaften, den Verlust an Habitaten, den überwiegend schlechten Erhaltungszustand der meisten Biotoptypen und den schlechten ökologischen und chemischen Zustand vieler Gewässer in Deutschland. ${ }^{4}$ Durch die da-

Dr. iur. Stefan Möckel

Helmholtz-Zentrum für Umweltforschung - UFZ,

Permoserstr. 15, 04318 Leipzig, Deutschland

Ass. jur., B. Sc. Geogr. André Wolf

Helmholtz-Zentrum für Umweltforschung - UFZ,

Permoserstr. 15, 04318 Leipzig, Deutschland mit einhergehende Überformung und Degradierung natürlicher Ökosysteme wurde die Vielfalt der Ökosystemleistungen zugunsten hoher land- und forstwirtschaftlicher Erträge reduziert. Die Ackerbaustrategie des Bundeslandwirtschaftsministeriums (BMEL) erkennt die ökologischen Probleme und auch den Handlungsbedarf ausdrücklich an. ${ }^{5}$ Flurbereinigungen haben einen wesentlichen Anteil an den Umgestaltungen der (Kultur-)Landschaft.

Das Instrument der Flurbereinigung ist das einzige staatliche Instrument, welches im ländlichen Raum rechtsverbindlich sowohl eine Neuordnung von Grundeigentum als auch eine Umgestaltung von Landschaften ermöglicht. Die Wirkungen für Umwelt, Natur und Ökosysteme können je nach Zielsetzung und abhängig von den konkret festgesetzten Maßnahmen negativ oder positiv sein. Die Bedeutung der Flurbereinigung als Instrument für den Erhalt von Ökosystemleistungen in Agrarlandschaften und Forsten ist in Anbetracht der rechtlichen Möglichkeiten groß, ${ }^{6}$ wird

^ Die Autoren danken Anne Wessner für das Lektorat und zwei anonymen Gutachtern für hilfreiche ergänzende Hinweise. Die Arbeit wurde mitfinanziert vom Bundesministerium für Bildung und Forschung (BMBF) im Rahmen der Förderinitiative „Boden als nachhaltige Ressource für die Bioökonomie - BonaRes“, Förderkennzeichen 031B0511A.

1) BfN, Daten zur Natur 2016, S. 50.

2) EEA, Agricultural land use intensity. Data and Maps, 2015.

3) Zu allen drei Punkten vgl. Poschlod, Geschichte der Kulturlandschaft. Stuttgart (Hohenheim) 2017, S. 115-183, 218-221; Heißenhuber/Haber/Krämer, 30 Jahre SRU - Sondergutachten „Umweltprobleme der Landwirtschaft'، - eine Bilanz, 2015, S. 176-183; Haber, Landwirtschaft und Naturschutz. Weinheim 2014, S. 8993; Küster, Geschichte der Landschaft in Mitteleuropa. München 2010, S. 279-287, 365-376

4) BMU/BFN, Die Lage der Natur in Deutschland, 2020; Heinze u.a., Natur und Landschaft 2019, 453, 453-462, https://doi.org/ 10.17433/11.2019.50153745; v.d. Decken u.a., Agrar-Report 2017 - Biologische Vielfalt in der Agrarlandschaft, BfN, Bonn 2017; BfN, Daten zur Natur 2016; UBA, Die Wasserrahmenrichtlinie: Deutschlands Gewässer 2015, 2016; Eichenberg u.a., Global Change Biology 2020, 1-14, https://doi.org/https://doi.org/ 10.1111/gcb.15447; Hallmann u.a., PLOS ONE 2017, 10.1371/ journal.pone.0185809, 21, https://doi.org/10.1371/journal.pone. 0185809; Hoffmann u.a., Bewertung und Verbesserung der Biodiversität leistungsfähiger Nutzungssysteme in Ackerbaugebieten unter Nutzung von Indikatorvogelarten. Braunschweig 2012; Wesche u.a., Biological Conservation 2012, 76, 76-85, https://doi.org/10.1016/j.biocon.2012.02.015; Wahl u.a., Vögel in Deutschland, Münster 2011; Kattwinkel u.a., Ecological Applications 2011, 2068, 2068-2081, https://doi.org/10.1890/10-1993.1. $\mathrm{Zu}$ den teilweise drastischen Rückgängen bei einzelnen Artengruppen in Agrarlandschaften vgl. Schuch u.a., Natur und Landschaft 2019, 141, 141-145; Wesche/Schuch/Bock, Natur und Landschaft 2014, 417, 417-421; Leuschner u.a., Natur und Landschaft 2014, 386, 386-391; Krause u. a., Natur und Landschaft 2014, 399, 399-404; Hötker u. a., Natur und Landschaft 2014, 410, 410-416; Meyer u.a., Diversity and Distributions 2013, 1175, 1175-1187, https://doi.org/10.1111/ddi.12102; Steffen u.a., Hydrobiologia 2013, 1, 1-17, https://doi.org/10.1007/s10750-013-1472-2.

5) BMEL, Ackerbaustrategie 2035 - Perspektiven für einen produktiven und vielfältigen Pflanzenbau, 2021, S. 20.

6) Arge Landentwicklung, Strategische Lösungsansätze und BestPractice-Beispiele zum Thema Landentwicklung und Naturschutz, Schriftenreihe Heft 24, 2016, S. 7-17, 30. 
allerdings oftmals nicht ausreichend gesehen. ${ }^{7}$ Umgekehrt wird bei der Flurbereinigung die Bedeutung von Okosystemen und ihrer Regulierungs- und Erhaltungsleistungen für die Land- und Forstwirtschaft unterschätzt. ${ }^{8}$

In der Vergangenheit dienten Flurbereinigungsverfahren dazu, die ehemals kleinteilige Kulturlandschaft an die neuen technischen Möglichkeiten der Land- und Forstwirtschaft anzupassen, um die Erträge zu steigern und die Arbeitskosten zu senken. Des Weiteren wurde mit dem Instrument die Bereitstellung von Land für Infrastrukturprojekte wie Autobahn- und Eisenbahntrassen ermöglicht. Bis 1976 war auch das Flurbereinigungsrecht in erster Linie ein Instrument zur Verbesserung der Produktions- und Arbeitsbedingungen in der Land- und Forstwirtschaft. ${ }^{9}$ Mit den immer sichtbareren Umweltfolgen ${ }^{10}$, der landwirtschaftlichen Überproduktion und den veränderten Ansprüchen an den ländlichen Raum wurden 1976 in der Bundesrepublik Deutschland die Zielsetzungen in $\$ 1$ Flurbereinigungsgesetz (FlurbG) um die Förderung der allgemeinen Landeskultur und der Landentwicklung erweitert und in $\$ \$ 37,86$, 91, 103a FlurbG die Belange des Umwelt- und Naturschutzes sowie der Landschaftspflege gestärkt. ${ }^{11}$

Im Ringen um das Verhältnis der drei Zielsetzungen zueinander wurden die Maßnahmen des Naturschutzes und der Landschaftspflege zunächst unter die einschränkende Prämisse der Notwendigkeit gestellt. ${ }^{12}$ Erst 1994 wurde die Prämisse der Novellierung in $\$ 86$ Abs. 1 Nr. 1 FlurbG aufgegeben, um noch besser Ziele des Naturschutzes und der Landschaftspflege mit der Flurbereinigung zu befördern und entstehende Landnutzungskonflikte schneller zu lösen. ${ }^{13}$ Gleichzeitig definierte der Gesetzgeber in $\$ 86$ Abs. 1 Nr. 1 FlurbG den Begriff, ,Landentwicklung“ nun dahingehend, dass er ,insbesondere Maßnahmen der Agrarstrukturverbesserung, der Siedlung, der Dorferneuerung, städtebauliche Maßnahmen, Maßnahmen des Umweltschutzes, der naturnahen Entwicklung von Gewässern, des Naturschutzes und der Landschaftspflege oder der Gestaltung des Orts- und Landschaftsbildes" umfasst. Auch der Begriff der „Landeskultur“ schließt nach heutigem Verständnis ökonomische und ökologische Aspekte mit ein und enthält damit auch den Auftrag zur Förderung der ökologischen Funktionen des ländlichen Raumes. ${ }^{14}$

Seitdem werden Flurbereinigungsverfahren außer zur Förderung der land- und forstwirtschaftlichen Produktionsbedingungen immer mehr auch zum Erhalt und zur Wiederherstellung von Landschaftselementen und Habitaten sowie zur Schaffung, Renaturierung und Sicherung von Ökosystemen, biologischer Vielfalt und ökologisch wertvollen Flächer genutzt. ${ }^{15}$ Dienen Flurbereinigungsverfahren jedoch v.a. der Verbesserung landwirtschaftlicher Produktionsbedingungen, kommt es auch heute noch zu Konflikten mit dem Natur- und Umweltschutz ${ }^{16}$, da eine Intensivierung der landwirtschaftlichen Bodennutzung regelmäßig mit einem Rückgang der biologischen Vielfalt verbunden ist. ${ }^{17}$

Staatlich organisierte Flurbereinigungsverfahren sind auch gegenwärtig wichtige planungsrechtliche Instrumente zur Neuordnung der Eigentumsverhältnisse und zur Umgestaltung der Landschaft im ländlichen Raum. Die Länder führen stetig eine Vielzahl an Flurbereinigungsverfahren durch und halten hierfür umfangreiche Verwaltungskapazitäten vor. ${ }^{18}$ Dabei gilt trotz der 2006 erfolgten Föderalismusreform ${ }^{19}$ das Flurbereinigungsrecht des Bundes gemäß Art. 125a Abs. 1 Grundgesetz (GG) fort, solange ein Bundesland für sein Gebiet die Flurbereinigung nicht umfassend selbst regelt. Das Flurbereinigungsgesetz trifft dabei hinsichtlich der Verfahren eine grundsätzliche Unterscheidung zwischen der privatnützigen Flurbereinigung, welche ländlichen Grundbesitz ${ }^{20}$ gemäß den Zielen des $\$ 1$ FlurbG neu ordnet, und der unternehmensbezogenen Flurbereinigung, welche gemäß $\$ 87$ FlurbG der Verwirklichung eines planfestgestellten Vorhabens mit zulässiger Enteignung dient und den damit verbundenen Verlust an land- und forstwirtschaftlichen Flächen auf eine größere Solidargemeinschaft verteilt. ${ }^{21}$

In den folgenden Abschnitten wird aufgezeigt, in welchem Umfang die verschiedenen Verfahren des Flurbereinigungsrechts die Belange des Umwelt- und Naturschutzes aufgreifen und inwieweit Maßnahmen zur Wiederherstellung von Ökosystemen und ihrer Leistungen privatnützige gemeinschaftliche Anlagen darstellen. Das Flurbereinigungsgesetz sieht verschiedene Verfahren vor, die in unterschiedlichem Umfang die Aufwertung und Wiederherstellung von Ökosystemen ermöglichen (siehe Abschnitt 2). Privatnützige Flurbereinigungsverfahren (insbesondere Regel- und vereinfachte Verfahren) müssen gemäß $₫ 4$ FlurbG dabei vorrangig den privaten Interessen der Teilnehmergemein-

7) Vgl. Leopoldina/acatech/Akademienunion, Biodiversität und Management von Agrarlandschaften - Umfassendes Handeln ist jetzt wichtig, 2020; WBBGR, Wie die Politik auf die Bedrohung der Biodiversität in Agrarlandschaften durch den Klimawandel reagieren kann, 2020; SRU/WBBGR, Für einen flächenwirksamen Insektenschutz, 2018.

8) Vgl. Arge Landentwicklung, Strategische Lösungsansätze und Best-Practice-Beispiele zum Thema Landentwicklung und Naturschutz, Schriftenreihe Heft 24, 2016, S. 24-30.

9) Thomas, NuR 2014, 618, 618-623; Thomas, NuR 2014, 686, 686-691; Weiß, Zur Entwicklung des Flurbereinigungsgesetzes der Bundesrepublik Deutschland in den vergangenen $6 \mathrm{Jahr}-$ zehnten, Butjadingen-Stollham 2009, S. 3-15. Auch in der DDR erfolgten in großem Umfang Flurbereinigungen.

10) Vgl. SRU Umweltgutachten 1974, S. 110-133; SRU Umweltgutachten 1978, S. 390-392, 412-419.

11) Bundesregierung, Entwurf eines Gesetzes zur Änderung des Flurbereinigungsgesetzes 1974, BT-Drs. 7/3020, S. 1-20; Thomas, NuR 2014, 686, 687-689; Weiß, Zur Entwicklung des Flurbereinigungsgesetzes der Bundesrepublik Deutschland in den vergangenen 6 Jahrzehnten, Butjadingen-Stollham 2009, S. 16-25.

12) Thomas, NuR 2014, 686, 687-688; Weiß, Zur Entwicklung des Flurbereinigungsgesetzes der Bundesrepublik Deutschland in den vergangenen 6 Jahrzehnten, Butjadingen-Stollham 2009, S. $20-23$.

13) Vgl. Bundesregierung, Entwurf eines Gesetzes zur Änderung des Flurbereinigungsgesetzes 1994, BT-Drs.12/7909, S. 6-8; Thomas, NuR 2014, 686, 689; Weiß, Zur Entwicklung des Flurbereinigungsgesetzes der Bundesrepublik Deutschland in den vergangenen 6 Jahrzehnten, Butjadingen-Stollham 2009, S. 31-39.

14) Seuffert, Die Flurbereinigung vor dem Hintergrund des Art. 14 GG, Freiburg 2011, S. 93-101; Thiemann, RdL 2017, 241, 242; Thomas, RdL 2014, 293, 294; Thomas, NuR 2014, 686, 689; Weiß, Zur Entwicklung des Flurbereinigungsgesetzes der Bundesrepublik Deutschland in den vergangenen 6 Jahrzehnten, Butjadingen-Stollham 2009, S. 19; Wingerter in: Wingerter/ Mayr, FlurbG, Standardkommentar, 10. Aufl., 2018, \$1 Rdnr. 3.

15) Arge Landentwicklung, Leitlinien Landentwicklung - Zukunft im ländlichen Raum gemeinsam gestalten - Orientierungsrahmen zur nachhaltigen Entwicklung der ländlichen Räume, 2011, S. 8, 15f.; Arge Landentwicklung, Strategische Lösungsansätze und Best-Practice-Beispiele zum Thema Landentwicklung und Naturschutz, Schriftenreihe Heft 24, 2016; Binder, Flurbereinigungsplanung und Ökosystemschutz als Rechts- und Governance-Problem, Marburg 2019, S. 62-64, 197-205; Oppermann u. a., Flurbereinigung und Naturschutz - Situation und Handlungsempfehlungen, Berlin 2003.

16) Oppermann u. a., Flurbereinigung und Naturschutz, Berlin 2003.

17) Vgl. Beckmann u. a., Global Change Biology 2019, 1941-1956, https://doi.org/https://doi.org/10.1111/gcb.14606.

18) Heißenhuber/Haber/Krämer, 30 Jahre SRU - Sondergutachten „Umweltprobleme der Landwirtschaft" - eine Bilanz, 2015, S. 100; Oppermann u.a., Flurbereinigung und Naturschutz, Berlin 2003, S. 4; Thomas, Flächenmanagement und Bodenordnung 2005, 179, 180-183; Thomas, Flächenmanagement und Bodenordnung 2009, 56 .

19) BT-Drs.16/813 2006, S. 12-13.

20) Für nicht ländlichen Grundbesitz gilt die Baulandumlegung nach $\$ \$ 45-84$ Baugesetzbuch.

21) Vgl. BVerwG, Urt. v. 13.4.2011 - 9 C 2.10, Rdnr. 13-21. 
schaft dienen, die aus Grundstückseigentümern und Erbbauberechtigten besteht. Diese Voraussetzung verhindert nach dem Bundesverfassungsgericht, dass diese Flurbereinigungsverfahren zu einer Enteignung werden, welche gemäß Art. 14 Abs. 3 Grundgesetz nur zum Wohle der Allgemeinheit und nur gegen eine angemessene Entschädigung zulässig ist. ${ }^{22}$ Maßnahmen des Umwelt- und Naturschutzes wurden bisher meist nicht als privatnützig angesehen, was den Anwendungsbereich des Instruments einschränkte. Unter 3 wird ausführlich untersucht, inwieweit Ökosystemleistungen die Interessen der Eigentümer und Nutzer von Grundstücken sichern oder befördern und Maßnahmen zu ihrem Erhalt oder ihrer Wiederherstellung als privatnützig einzustufen sind. Die Ergebnisse werden in 4 zusammengefasst und um weitergehende politische Empfehlungen ergänzt.

\section{Verfahrensarten}

Nach dem Flurbereinigungsgesetz gibt es fünf unterschiedliche Flurneuordnungsverfahren:

- die Regelflurbereinigung nach $\$ 1$ FlurbG,

- die vereinfachte Flurbereinigung nach $\$ 86$ FlurbG,

- die Unternehmensflurbereinigung nach $\$ 87$ FlurbG,

- das beschleunigte Zusammenlegungsverfahren nach $§ 91$ FlurbG und

- den freiwilligen Landtausch gemäß \103a FlurbG.

Die Regelungen zur privatnützigen Regelflurbereinigung erstrecken sich über das gesamte Gesetz mit Ausnahme der vier besonderen Verfahren. Die vereinfachte Flurbereinigung und das beschleunigte Zusammenlegungsverfahren sind verfahrensregulatorisch reduzierte Varianten der Regelflurbereinigung. Die fremdnützige Unternehmensflurbereinigung und auch der freiwillige Landtausch stellen Sonderverfahren dar, bei denen allerdings etliche Verfahrensvorschriften des Regelverfahrens Anwendung finden.

\subsection{Regelflurbereinigung}

Die Regelflurbereinigung beinhaltet ein mehrstufiges Verfahren, welches regelmäßig mehrere Jahre andauert. ${ }^{23}$ Eine Flurbereinigung beginnt mit dem Anordnungsbeschluss einer oberen Flurbereinigungsbehörde gemäß \4 FlurbG, in dem u.a. auch das Flurbereinigungsgebiet festgelegt ist. Dabei hat die Behörde vor dem Anordnungsbeschluss nach \$S 5 Abs. 1 und 2, 10 Abs. 1 FlurbG eine Aufklärung der zu beteiligenden Grundstückseigentümer und Erbbauberechtigten (Teilnehmer) sowie eine Anhörung der landwirtschaftlichen Berufsvertretung, der Landesplanungsbehörde, der Gemeinden und sonstiger zu beteiligender Organisationen und Behörden durchzuführen. Die obere Flurbereinigungsbehörde muss ein Flurbereinigungsverfahren zur Erreichung der Ziele und Zwecke in $\$ \$ 1$ und 37 FlurbG für erforderlich erachten und es darf kein einfacheres und somit milderes Verfahren, wie z. B. das beschleunigte $\mathrm{Zu}$ sammenlegungsverfahren oder der freiwillige Landtausch, möglich sein. ${ }^{24}$ Weiterhin muss sie die Privatnützigkeit des Verfahrens prüfen und begründen.

Dem Anordnungsbeschluss folgen das Wertermittlungsverfahren nach \$\$27-33 FlurbG sowie die planerische Neugestaltung des Flurbereinigungsgebietes gemäß $\quad$ S\$ 37 Abs. 1 S. 2, 40 FlurbG mittels Flurbereinigungsplan und ergänzendem Wege- und Gewässerplan. Nach \$37 Abs. 1 FlurbG ist das Flurbereinigungsgebiet unter Beachtung der jeweiligen Landschaftsstruktur so neu zu gestalten, wie es den gegeneinander abzuwägenden Interessen der Beteiligten sowie den Interessen der allgemeinen Landeskultur und der Landentwicklung entspricht und wie es das Wohl der Allgemeinheit erfordert. Im Rahmen dieser planerischen Abwägung sind gemä $\$ 37$ Abs. 2 FlurbG explizit die Belange des Umwelt- und Naturschutzes, der Landschaftspflege sowie der Erholung zu berücksichtigen. ${ }^{25}$ Die Neugestaltung umfasst nach $₫ 37$ Abs. 1 FlurbG insbesondere:
- die Neueinteilung der Feldmark durch die Zusammenlegung zersplitterter oder unwirtschaftlich geformter Grundstücke,

- die Schaffung von Wegen, Straßen, Gewässern und anderen gemeinschaftlichen Anlagen,

- die Durchführung bodenschützender und bodenverbessernder sowie landschaftsgestaltender Maßnahmen, wobei Letztere u.a. auch die Wiederherstellung von Landschaftselementen oder die Herstellung von Biotopverbundsystemen als autonome Gestaltungsaufgabe der Flurbereinigungsbehörden mit einschließen, ${ }^{26}$

- sonstige Maßnahmen zur Verbesserung der wirtschaftlichen Grundlagen, Verminderung des Arbeitsaufwandes und Erleichterung der Bewirtschaftung,

- die Ordnung der rechtlichen Verhältnisse,

- fakultative Maßnahmen der Dorferneuerung sowie

- die Bereitstellung von Land für öffentliche Anlagen zu Gunsten der Allgemeinheit.

Im Flurbereinigungsplan werden gemäß $₫ 58$ FlurbG die zukünftige Gestaltung des Gebietes in rechtlicher und tatsächlicher Hinsicht festgelegt und die Ergebnisse des gesamten Verfahrens zusammengefasst. Er ist eine Allgemeinverfügung ${ }^{27}$, mit der auch andere bestehende Rechte (z.B. wasserrechtliche Gestattungen, Unterhaltspflichten, Grunddienstbarkeiten oder Erschließungsbeitragspflichten) geändert werden können ${ }^{28}$, sofern dies den Zielen des $\$ 1$ FlurbG dient und im FlurbG eine konkrete Einzelermächtigung für die betreffende Maßnahme existiert. ${ }^{29}$ Für Festsetzungen, die im gemeinschaftlichen Interesse der Beteiligten oder im öffentlichen Interesse getroffen werden, hat er nach $\int 58$ Abs. 4 FlurbG die Wirkung einer Gemeindesatzung.

Der Wege- und Gewässerplan mit landschaftspflegerischem Begleitplan legt gemäß \$41 Abs. 1 FlurbG die Anlagen im gemeinschaftlichen Interesse der Teilnehmer $(\mathbb{S} 39$ FlurbG) und im öffentlichen Interesse ( $\$ 40$ FlurbG) einschließlich wasserwirtschaftlicher, bodenverbessernder sowie landschaftsgestaltender Maßnahmen fest. Zu den gemeinschaftlichen Anlagen gehören auch ökologisch relevante Maßnahmen wie z.B. die Melioration, die Veränderung der Boden- oder hydrologischen Verhältnisse (vgl. \37 Abs. 1 FlurbG) sowie die Ausgleichs- und Ersatzmaßnahmen, welche für die im Rahmen der Flurbereinigung erfolgenden Eingriffe gemäß $\ 15$ Bundesnaturschutzgesetz (BNatSchG) erforderlich sind. ${ }^{30}$ Die Teilnehmer sind nach $\$ S 42,47$ FlurbG verpflichtet, das Land für gemeinschaftliche Anlagen bereitzustellen sowie diese zu errichten und zu unterhalten bzw. die Herstellungs- und Unterhaltungskosten zu tragen, sofern nichts anderes festgelegt ist. Hierfür bilden alle Teilnehmer nach $\$ 16$ FlurbG kraft Gesetzes eine Körperschaft des öffentlichen Rechts (Teilnehmergemeinschaft).

Öffentliche Anlagen sind alle Anlagen und Maßnahmen im öffentlichen Interesse (z.B. dem öffentlichen Verkehr

22) BVerfG, Urt. v. 31.1.2001 - 6 CN 2/00, Rdnr. 29-32, NVwZ 2001, 1035

23) Vgl. Grimm, Agrarrecht, München 2004, S. 324-327.

24) Wingerter in: Wingerter/Mayr, FlurbG, Standardkommentar, 10. Aufl., 2018, $\$ 4$ Rdnr. 4

25) Binder, Flurbereinigungsplanung und Ökosystemschutz als Rechts- und Governance-Problem, Marburg 2019, S. 313-326.

26) Binder, Flurbereinigungsplanung und Ökosystemschutz als Rechts- und Governance-Problem, Marburg 2019, S. 311 f.; Thomas, NuR 2015, 98, 101; Wingerter in: Wingerter/Mayr, FlurbG, Standardkommentar, 10. Aufl., 2018, $\$ 37$ Rdnr. 18.

27) BVerwG, Urt. v. 20.5.1998 - 11 C 7.97, RdL 1998, 236, 237; Wingerter in: Wingerter/Mayr, FlurbG, Standardkommentar, 10. Aufl., 2018, $₫ 58$ Rdnr. 2.

28) Wingerter in: Wingerter/Mayr, FlurbG, Standardkommentar, 10. Aufl., 2018, $\$ 37$ Rdnr. 28-30.

29) BVerwG, Urt. v. 14. 6.1972 - V C 1/72, Rdnr. $145 \mathrm{f}$

30) Möckel, UPR 2012, 247, 254. 
gewidmete Straßen und Gewässer oder die Ausweișung von Schutzgebieten oder Biotopverbundflächen). Über den gegebenenfalls eingebrachten Anteil öffentlicher Flächen hinaus können nach $₫ 40$ FlurbG nur in ,,verhältnismäßig geringem Umfange" weitere Flächen zur Verfügung gestellt werden; für mehr bedarf es einer Unternehmensflurbereinigung. Soweit eine Anlage nicht zugleich dem wirtschaftlichen Interesse der Teilnehmer dient, hat der Eigentümer der Anlage für das Land und entstehende Schäden einen angemessenen Kapitalbetrag an die Teilnehmergemeinschaft zu leisten.

Rechtlich ist die Erstellung des Wege- und Gewässerplans gemäß $\int 41$ Abs. 3 und 4 FlurbG ein Planfeststellungsverfahren. Seine Aufstellung hat nach $₫ 41$ Abs. 1 FlurbG im Benehmen mit dem Vorstand der Teilnehmergemeinschaft zu erfolgen, was zwar eine enge Abstimmung, aber kein Einvernehmen erfordert. ${ }^{31}$ Gemäß S\$63 Abs. 2 Nr. 6 und 7, 64 Abs. 1 BNatSchG ist anerkannten Naturschutzverbänden Gelegenheit zur Einsicht und Stellungnahme zu geben und diese können die Verletzung von Naturschutzrecht auch gerichtlich geltend machen. Dem Planfeststellungsbeschluss kommt nach $\$ 41$ Abs. 5 FlurbG i.V.m. $\$ \$ 75$ Abs. 1, 74 Abs. 6 Verwaltungsverfahrensgesetz eine rechtliche Konzentrationswirkung $\mathrm{zu}$, weshalb mit ihm andere Genehmigungen ersetzt oder abgeändert werden können (z. B. naturschutzrechtliche Gestattungen, Baugenehmigungen, wasserrechtliche Erlaubnisse und auch Planfeststellungsverfahren zum Gewässerausbau), sofern dies den Zielen der Flurbereinigung dient. ${ }^{32}$

\subsection{Vereinfachte und beschleunigte Verfahren}

Sofern ein höheres öffentliches Interesse an einer Flurbereinigung besteht (z.B. Landentwicklung, Dorferneuerung, Umweltschutz), kann nach $\$ 86$ FlurbG ein vereinfachtes Flurbereinigungsverfahren durchgeführt werden ${ }^{33}$, bei dem u.a. die Beteiligungs- und Berücksichtigungspflichten reduziert sind und auch die Aufstellung eines gesonderten Wege- und Gewässerplanes mit landschaftspflegerischem Begleitplan fakultativ ist. Nach $₫ 86$ Abs. 1 Nr. 1 FlurbG ist das vereinfachte Verfahren auch für Maßnahmen des Umweltschutzes, der naturnahen Entwicklung von Gewässern, des Naturschutzes und der Landschaftspflege oder zur Gestaltung des Orts- und Landschaftsbildes einsetzbar. Allerdings bleibt auch die vereinfachte Flurbereinigung ein privatnütziges Verfahren, weshalb die privaten Interessen weiterhin die öffentlichen Interessen überwiegen müssen und das Verfahren nicht allein zur Landbeschaffung für Interessen der Allgemeinheit eingesetzt werden darf. ${ }^{34}$ Aufgrund des höheren öffentlichen Interesses und der reduzierten Beteiligungsrechte der Teilnehmer sind der Teilnehmergemeinschaft nach $\$ 86$ Abs. 3 FlurbG die Ausführungskosten für die Maßnahmen nach Absatz $1 \mathrm{zu}$ erstatten.

Um die mit der Flurbereinigung angestrebte Verbesserung der Produktions- und Arbeitsbedingungen in der Land- und Forstwirtschaft möglichst rasch herbeizuführen oder um notwendige Maßnahmen des Naturschutzes und der Landschaftspflege zu ermöglichen, kann in Gemarkungen, in denen die Anlage eines neuen Wegenetzes und größere wasserwirtschaftliche Maßnahmen zunächst nicht erforderlich sind, eine Flurbereinigung nach dem „,beschleunigten Zusammenlegungsverfahren“ gemäß SS 91103 FlurbG erfolgen. Die Naturschutzbehörden können das Verfahren nach $\$ 93$ Abs. 1 FlurbG beantragen, wenn die Zusammenlegung zugleich auch dem Interesse der betroffenen Grundstückseigentümer dient. Die Maßnahmen des Naturschutzes und der Landschaftspflege sind im Zusammenlegungsplan darzustellen, der sowohl den Flurbereinigungsplan als auch den Wege- und Gewässerplan ersetzt ( $\$ 97$ FlurbG).

\subsection{Unternehmensflurbereinigung}

Benötigt ein planfeststellungspflichtiges Vorhaben, für welches eine enteignungsrechtliche Vorwirkung gesetzlich vorgesehen ist (z.B. der Bau von Straßen, Eisenbahnen, Wasserstraßen, Flughäfen, aber auch Gewässerausbauten oder Hochwasserschutzanlagen), ländliche Grundstücke in größerem Umfang, ist gemäß $§ 87$ Abs. 1 FlurbG die Unternehmensflurbereinigung einschlägig. ${ }^{35}$ Diese ist wegen der überwiegenden Fremdnützigkeit ein Enteignungsverfahren i.S.v. Art. 14 Abs. 3 GG. ${ }^{36}$ Für das in Anspruch genommene Land sieht $₫ 88 \mathrm{Nr} .4$ und 5 FlurbG statt einer Landabfindung lediglich eine Geldentschädigung vor. Allerdings umfasst die Unternehmensflurbereinigung selbst keine Enteignungsermächtigung, sondern ist lediglich ein Durchführungsverfahren, um die Lasten und negativen Auswirkungen einer fachgesetzlich zulässigen Enteignung (z. B. für Gewässer- und Deichbauten nach \$ 71 Wasserhaushaltsgesetz - WHG oder Fernstraßen gemäß $₫ 18 f$ Bundesfernstraßengesetz) auf einen größeren Kreis von Eigentümern zu verteilen. Dies soll übermäßige Belastungen Einzelner, Existenzgefährdungen landwirtschaftlicher Betriebe und Nachteile für die allgemeine Landeskultur vermeiden. ${ }^{37}$ Sofern fachgesetzlich nur eine Nutzungsbeschränkung, aber keine Enteignung vorgesehen ist (so derzeit bundesrechtlich bei der Ausweisung von naturschutz- oder wasserrechtlichen Schutzgebieten), kommt die Unternehmensflurbereinigung nicht in Betracht. ${ }^{38}$ Allerdings könnten der Bund oder gemäß Art. 72 Abs. 2 Nr. 2 und 5 GG auch die Länder entsprechende Planfeststellungsverfahren mit enteignungsrechtlicher Vorwirkung im Sinne von Art. 14 Abs. 3 GG für Schutzgebiete oder Biotopverbindungen vorsehen, sofern es zum Wohle der Allgemeinheit erforderlich ist. ${ }^{39}$

\subsection{Freiwillige Bereinigungsmaßnahmen}

Auch der freiwillige Landtausch nach $₫ 103$ a FlurbG dient der beschleunigten Verbesserung der Agrarstruktur, kann aber nach Absatz 2 auch aus Gründen des Naturschutzes und der Landschaftspflege erfolgen. Gegenüber dem privaten Nutzungstausch nach $\$ 480$ BGB ist der Landtausch immer noch ein behördlich geleitetes Verfahren, bei dem auch konkurrierende öffentliche Belange (z. B. des Umwelt- und Naturschutzes) mit zu berücksichtigen sind. ${ }^{40}$ Die Durchführung erfordert allerdings das Einverständnis aller betroffenen Eigentümer, weshalb in der Praxis das Verfahren

31) Wingerter in: Wingerter/Mayr, FlurbG, Standardkommentar, 10. Aufl., 2018, \41 Rdnr. 9.

32) Wingerter in: Wingerter/Mayr, FlurbG, Standardkommentar, 10. Aufl., 2018, \$41 Rdnr. 1, 16-24ff., $31 \mathrm{f}$.

33) BVerwG, Beschl. v. 18.6.1998 - 11 B 28.98, RdL 1998, 209, $209 \mathrm{f}$.

34) BVerwG, Urt. v. 13. 4.2011 - 9 C 2.10, Rdnr. 15-21.

35) BVerwG, Urt. v. 13. 4.2011 - 9 C 2.10, Rdnr. 14.

36) BVerfG, Urt. v. 24.3.1987 - 1 BvR 1046/85, Rdnr. 280; BVerwG, Urt. v. 29.1.2009 - 9 A 3.08, Rdnr. 17.

37) Vgl. $\$ 87$ Abs. 1 FlurbG; BVerfG, Urt. v. 24.3.1987 - 1 BvR 1046/85, Rdnr. 281 f.; BVerwG, Urt. v. 21.10.2009 - 9 C 9.08 , NuR 2010, 411, 413; Weiß, RdL 2006, 141, 142-145; Wingerter in: Wingerter/Mayr, FlurbG, Standardkommentar, 10. Aufl., 2018, Vorb. zu $\$ 87$, Rdnr. 2 f.

38) BVerwG, Beschl. v. 17.1.2000 - 6 BN 2.99, NuR 2000, 276; BVerwG, Urt. v. 31.1.2001 - 6 CN 2/00, NVwZ 2001, 1035, 1036. Nur für bestimmte Einzelmaßnahmen ist gemäß $₫ 68$ Abs. $3 \mathrm{BNatSchG}$ landesrechtlich eine Enteignung vorgesehen (z.B. Art. 40 BayNatSchG 2011; $\$ 37$ SächsNatSchG 2011).

39) Binder, Flurbereinigungsplanung und Ökosystemschutz als Rechts- und Governance-Problem, Marburg 2019, S. 379-384; vgl. Thomas, NuR 2015, 98, 102.

40) Kritisch zu diesem „Instrument“ Oppermann u.a., Flurbereinigung und Naturschutz, Berlin 2003, S. 10. 
überwiegend zur Verbesserung der Produktionsbedingungen eingesetzt wird und gemeinschaftliche oder öffentliche Anlagen nur selten festgesetzt werden. Aus Gründen des Naturschutzes und der Landschaftspflege erfolgt der Landtausch daher v.a., wenn öffentlich-rechtliche Körperschaften und Naturschutzorganisationen Flächen tauschen wollen.

\section{3. Ökosystemleistungen und privatnützige Flurbereinigungsverfahren}

Eigentum ist ein rechtliches Konstrukt, weshalb gemäß Art. 14 Abs. 1 GG auch sein Inhalt erst durch das Recht bestimmt wird. Daher ist nur der vollständige oder teilweise Entzug einer durch das Recht eingeräumten Eigentümerposition zugunsten öffentlicher Aufgaben eine Enteignung im Sinne von Art. 14 Abs. 3 GG. ${ }^{41}$ Ist mit dem Entzug bestehender Rechtspositionen nur ein Ausgleich privater Interessen beabsichtigt, handelt es sich um eine Inhalts- und Schrankenbestimmung. ${ }^{42}$

Alle Flurbereinigungsverfahren mit Ausnahme der Unternehmensflurbereinigung sind keine Enteignungsverfahren, sondern Inhalts- und Schrankenbestimmungen im Sinne von Art. 14 Abs. 1 und 2 GG, da sie nach $\int S 4$, 10 Abs. 1 FlurbG vorrangig den Interessen der beteiligten Grundstückseigentümer und Erbbauberechtigten dienen (Privatnützigkeit) sowie die Grundflächen gemäß $₫ 44$ Abs. 1 FlurbG unter Berücksichtigung der eingebrachten Werte neu verteilt werden, ohne einen endgültigen Landentzug zu bezwecken. ${ }^{43}$ Sofern ein Flurbereinigungsverfahren nicht ausreichend die Privatnützigkeit und den wertmäßigen Landerhalt sicherstellt, schlägt es nicht in eine Enteignung nach Art. 14 Abs. 3 GG um, sondern ist rechtswidrig. ${ }^{44}$

Bevor untersucht wird, ob und wie der Erhalt bzw. die Wiederherstellung von Ökosystemen und ihrer Funktionen und Leistungen als privatnützig einzustufen ist, bedarf es einer genaueren Bestimmung von Privatnützigkeit.

\subsection{Privatnützigkeit}

Die Privatnützigkeit eines Flurbereinigungsverfahrens richtet sich weder nach der Zustimmung aller betroffenen Rechtsinhaber wie beim freiwilligen Landtausch noch nach der Mehrheitsmeinung der Grundstückseigentümer und Erbbauberechtigten (Teilnehmer). ${ }^{45}$ Nach dem Bundesverwaltungsgericht (BVerwG) ist allein das objektive Interesse der Teilnehmergemeinschaft entscheidend. ${ }^{46}$ Dieses ist von der oberen Flurbereinigungsbehörde bei der Anordnung des Verfahrens anhand objektiver Erkenntnisse hinsichtlich der wohlverstandenen Erwartungen der Teilnehmer an eine zweckmäßige Flurneuordnung im Gebiet $\mathrm{zu}$ ermitteln und $\mathrm{zu}$ begründen. Das objektive Interesse muss sich zugleich innerhalb des gesetzlichen Rahmens bewegen, weshalb Interessen ausscheiden, die z. B. dem nationalen- oder europäischen Umwelt- und Naturschutzrecht widersprechen. ${ }^{47}$

Da Flurbereinigungsverfahren die Grundstücke im Gebiet für einen längeren Zeitraum neu ordnen und umgestalten (i.d. R. für mehrere Jahrzehnte), kommt den langfristigen Interessen der Teilnehmergemeinschaft und ihrer prognostischen Ermittlung durch die Flurbereinigungsbehörde eine hohe Relevanz zu. ${ }^{48}$ Soweit das objektive Interesse v.a. auf betriebswirtschaftliche Ziele der Teilnehmer reduziert wird ${ }^{49}$, verharrt dies bei der Rechtslage und Rechtsprechung ${ }^{50}$ vor der 1976 er Novellierung, als Flurbereinigungsverfahren nur für Agrarstrukturverbesserungen im Sinne einer Verbesserung der Produktions- und Arbeitsbedingungen in der Land- und Forstwirtschaft vorgesehen waren. ${ }^{51}$ Dies negiert den Umstand, dass sowohl der einzelne Teilnehmer als auch die Gesamtheit der Teilnehmer nicht nur ökonomische, sondern auch soziale und ökologische Interessen haben, wie die 1976 erweiterte Zielsetzung in $\$ 1$ FlurbG verdeutlicht. Ländliche Räume weisen eine Vielzahl von Landnutzungen auf (z. B. Erzeugung von Wind- und Solarstrom, Tourismus und Erholung) und immer mehr Grundeigentümer sind selbst keine aktiven Land- oder Forstwirte, sondern nur noch Verpächter. Hinzu kommt, dass die öffentliche Hand regelmäßig in Flurbereinigungsgebieten Grundstücke hält, weshalb auch diesbezügliche öffentliche Interessen bei der Bestimmung des objektiven Interesses mit einzubeziehen sind.

Da das Erfordernis der Privatnützigkeit in Flurbereinigungsverfahren (Ausnahme: Unternehmensflurbereinigung) aus der Eigentumsgarantie des Art. 14 Abs. 1 GG folgt, sind bei der Auslegung des Flurbereinigungsgesetzes und seiner Anwendung die höchstrichterliche Rechtsprechung zum Grundeigentum und seiner gesetzgeberischen Inhalts- und Schrankenbestimmung zu beachten. Die Rechtsprechung berücksichtigt, dass Grund und Boden sowohl unvermehrbar und situationsgebunden als auch in Ökosysteme eingebunden sind. Der ökonomische Wert und die private Nutzbarkeit von Grundeigentum sind abhängig von der Lage sowie den räumlichen, geografischen und klimatischen Gegebenheiten. Nach dem Bundesverfassungsgericht (BVerfG) ist daher das Grundeigentum im Hinblick auf seine funktionsgerechte Nutzung gemäß Art. 14 Abs. 2 GG im besonderen Maß der Sozialpflichtigkeit unterworfen und verbietet es die Unvermehrbarkeit von Grund und Boden, seine Nutzung dem freien Spiel der Kräfte und dem Belieben des Einzelnen vollständig zu überlassen. ${ }^{52}$ Das Bundesverwaltungsgericht betont weiter, dass jedes Grundstück durch seine Lage und Beschaffenheit sowie die Einbettung in seine Umwelt, also durch seine jeweilige Situation geprägt ist und dies auf die Eigentümerstellung ausstrahlt: „Wenn die natürlichen oder landschafts-

41) BVerfG, Beschl. v. 22.5.2001 - 1 BvR 1512/97 und 1677/97, Rdnr. 30.

42) BVerfG, Beschl. v. 22.5.2001 - 1 BvR 1512/97 und 1677/97, Rdnr. 30.

43) St. Rspr. BVerwG, Urt. v. 13.4.2011 - 9 C 2.10, Rdnr. 14; BVerwG, Urt. v. 6.10.1960 - I C 64.60; vgl. auch BVerfG, Beschl. v. 22.5.2001 - 1 BvR 1512/97 und 1677/97, Rdnr. 29-32; BVerwG, Beschl. v. 9.11.1954 - I B 145.53, Rdnr. 227f.; Wingerter in: Wingerter/Mayr, FlurbG, Standardkommentar, 10. Aufl., 2018, Vorb. zu $\$ 1$ Rdnr. 2; Seuffert, Die Flurbereinigung vor dem Hintergrund des Art. 14 GG, Freiburg 2011, S. 42-68.

44) Vgl. BVerfG, Beschl. v. 22.5.2001 - 1 BvR 1512/97 und 1677/97, Rdnr. 33-38; BVerfG, Beschl. v. 15.7.1981 - 1 BvL 77/78, Rdnr. 320; Weiß, RdL 2019, 162, 162-166.; Wingerter in: Wingerter/Mayr, FlurbG, Standardkommentar, 10. Aufl., 2018, Vorb. zu $\$ 1$ Rdnr. 4.

45) BVerwG, Beschl. v. 9.3.1990 - 5 B 78.89; BVerwG, Urt. v. 29.3.1968 - IV C 104.65, Rdnr. 259; BVerwG, Urt. v. 3.3.1959 - I C 142.56, Rdnr. 199; Wingerter in: Wingerter/Mayr, FlurbG, Standardkommentar, 10. Aufl., 2018, $\$ 4$ Rdnr. 5 f. m.w. N.

46) BVerwG, Beschl. v. 8.5.2019 - 9 B 19.18, Rdnr. 3; BVerwG, Beschl. v. 20.9.2019 - 9 B 50.18, Rdnr. 5; BVerwG, Urt. v. 13.4.2011 - 9 C 2.10, Rdnr. 13f.; BVerwG, Beschl. v. 9.3.1990 - 5 B 78.89; BVerwG, Beschl. v. 26.3.1974 - V B 14.72, Rdnr. 115f.; BVerwG, Urt. v. 29.3.1968 - IV C 104.65, Rdnr. 258f.; BVerwG Urt. v. 3.3. 1959 - I C 142.56, Rdnr. 199. 47) Thomas, NuR 2015, 98, 100.

48) Vgl. BVerwG, Beschl. v. 26.3.1974 - V B 14.72, Rdnr. 116; Möckel, UPR 2012, 247, 253.

49) So in BVerwG, Beschl. v. 9.3.1990 - 5 B 78.89; OVG Lüneburg, Beschl. v. 14. 10.2016 - 15 MF 8/16, Rdnr. 27; Hoecht, RdL 1991, $225,226$.

50) Z.B. BVerwG, Beschl. v. 26.3.1974 - V B 14.72, Rdnr. 115 f.

51) Binder, Flurbereinigungsplanung und Ökosystemschutz als Rechts- und Governance-Problem, Marburg 2019, S. 250-252; vgl. BVerwG, Beschl. v. 18.6.1998 - 11 B 28.98, RdL 1998, 209.

52) BVerfG, Beschl. v. 22.5.2001 - $1 \mathrm{BvR}$ 1512/97 und 1677/97, Rdnr. 37; BVerfG, Beschl. v. 12.1.1967 - 1 BvR 169/63, Rdnr. $82 \mathrm{f}$. 
räumlichen Gegebenheiten eines Grundstücks im Interesse der Allgemeinheit erhaltenswert sind und des Schutzes bedürfen, so ergibt sich hieraus eine Art immanente, d. h. dem Grundstück selbst anhaftende Beschränkung der Eigentümerbefugnisse, die durch natur- und landschaftsschutzrechtliche Regelungen lediglich nachgezeichnet wird.".53 Dementsprechend ist die von Art. 14 GG geschützte Privatnützigkeit bei Grund und Boden gemäß Art. 14 Abs. 2 GG in erheblichem Maße übergeordneten Allgemeinwohlinteressen verpflichtet. ${ }^{54}$

Die Einstufung von Interessen als privatnützig ist nicht nur wichtig für die Zulässigkeit eines privatnützigen Flurbereinigungsverfahrens, sondern auch entscheidend für die Frage, ob eine Maßnahme eine gemeinschaftliche Anlage im Sinne von $\$ 39$ FlurbG ist, für die gemäß $\$ \$ 42$, 47 FlurbG die Teilnehmergemeinschaft Land und Finanzmittel oder Arbeitsleistungen bereitstellen muss. Abgrenzungskriterium zu den öffentlichen Anlagen ist auch hier, dass die Anlage dem gemeinschaftlichen Interesse der Teilnehmer dient. Als privatnützig wurden bisher allgemein anerkannt:

- die Verbesserung der Produktionsbedingungen für die Land- und Forstwirtschaft (z. B. Melioration, maschinengerechte Gestaltung der Schläge und der Grundwasserstände) einschließlich Maßnahmen zum Erhalt oder zur Verbesserung von Böden ${ }^{55}$

- die Verbesserung der Grundstückszuschnitte (insbesondere die Zusammenlegung von durch Erbteilung entstandenen Splittergrundstücken) und

- Maßnahmen der Landentwicklung und zur Auflösung oder Abmilderung von Landnutzungskonflikten, bei denen rechtliche oder faktische Beschränkungen für die Teilnehmer sowie der land- und forstwirtschaftlichen Nutzung - z. B. aufgrund von Infrastrukturvorhaben oder Schutzgebietsausweisungen - durch eine Neuordnung oder sonstige integrierte Entwicklung des Gebietes abgemildert oder beseitigt werden, indem z. B. Flächen der öffentlichen Hand in die Schutzgebiete verlegt werden (Arrondierung) ${ }^{56}$

Insgesamt sind landentwickelnde Flurbereinigungsmaßnahmen, welche die Wohn-, Wirtschafts- und Erholungsfunktion erhalten oder verbessern und damit die Lebensverhältnisse im betreffenden Gebiet dauerhaft sichern oder aufwerten, im objektiven, wohlverstandenen und vorrangigen Interesse der Grundeigentümer und daher privatnützig. ${ }^{57}$

\subsection{Erhalt und Wiederherstellung von Ökosystemen und ihrer Leistungen}

Fraglich ist, ob und inwieweit der Erhalt bzw. die Wiederherstellung von Ökosystemen und ihrer Funktionen und Leistungen und damit der Schutz des Naturhaushaltes, der biologischen Vielfalt, des Klimas, der Gewässer und der Böden im objektiven Interesse der Teilnehmer steht. Das Flurbereinigungsgesetz ist hier uneindeutig. Einerseits nennt $\$ 40$ FlurbG entsprechende Schutzmaßnahmen als Beispiele für öffentliche Anlagen, wobei hier die Teilnehmergemeinschaft nach $₫ 40$ S. 2 FlurbG die Kosten übernehmen muss, wenn diese auch in ihrem Interesse sind. Andererseits führt $\$ \$ 37$ Abs. 1, 86 Abs. 1 Nr. 1 FlurbG dies auch als Aufgaben eines privatnützigen Flurbereinigungsverfahrens an (siehe Abschnitte 2.1 und 2.2). Die gesetzgeberischen Einordnungsschwierigkeiten spiegeln sowohl die Bedeutung der Natur und der Ökosysteme für die Gesellschaft und den einzelnen Grundstückseigentümer als auch den diesbezüglichen Erkenntniszuwachs wider.

Diese Bedeutung wurde mit dem Konzept der Ökosystemleistungen seit den neunziger Jahren wissenschaftlich herausgearbeitet. ${ }^{58}$ Die Bedeutung der Leistungs- und Funktionsfähigkeit der Ökosysteme einschließlich ihres Erholungswertes wird rechtlich unter anderem von $₫ 1$ Abs. 1 Nr. 2 und 3, Abs. 3 und 4 BNatSchG anerkannt, wo- bei der Gesetzgeber statt Ökosysteme den Begriff Naturhaushalt verwendet. ${ }^{59}$ Bei den Ökosystemleistungen wird allgemein unterschieden zwischen Versorgungsleistungen, Regulierungs- und Erhaltungsleistungen sowie kulturellen Leistungen. Die land- und forstwirtschaftliche Primärproduktion von Nahrungs- und Futtermitteln sowie von Rohstoffen für die Produktion und die Energiegewinnung hat in dem Konzept eine besondere Stellung. Zum einen wird sie als Versorgungsleistung und damit als Ökosystemleistung eingestuft ${ }^{60}$, obwohl sie insbesondere bei der industriellen Landwirtschaft in erheblichem Maße auch auf künstlichen Faktoren (u. a. Einsatz von Dünge- und Pflanzenschutzmitteln, Maschinen, gezüchtetem oder genetisch verändertem Saatgut und ebensolchen Nutztierarten) beruht. ${ }^{61}$ Zum anderen ist sie selbst in hohem Maße auf die Regulierungs- und Erhaltungsleistungen von Ökosystemen angewiesen (siehe Tabelle). Diese Leistungen bieten für die Grundstückseigentümer bzw. die land- und forstwirtschaftlichen Bewirtschafter geldwerte Vorteile, welche den Gebrauchswert und demzufolge auch den Marktwert der Flächen erhöhen. ${ }^{62}$

Gleichzeitig beeinträchtigen intensive land- und forstwirtschaftliche Bewirtschaftungsformen die Regulierungs- und Erhaltungsleistungen, indem z.B. Bodenprozesse gestört werden und die Bodenerosion gefördert wird, Habitate und Habitateigenschaften für wildlebende Tierund Pflanzenarten und damit für viele Nützlinge reduziert werden oder sowohl das globale als auch das regionale und

53) BVerwG, Urt. v. 24.6.1993 - 7 C 26.92, Rdnr. 4.

54) Vgl. Binder, Flurbereinigungsplanung und Ökosystemschutz als Rechts- und Governance-Problem, Marburg 2019, S. 219-240.

55) Vgl. Seuffert, Die Flurbereinigung vor dem Hintergrund des Art. 14 GG, Freiburg 2011, S. 89-96.

56) Vgl. Bundesregierung, Entwurf eines Gesetzes zur Änderung des Flurbereinigungsgesetzes 1994, BT-Drs.12/7909, S. 6-8; BVerwG, Beschl. v. 18.11.2014 - 9 B 30.14, Rdnr. 4f.; BVerwG, Urt. v. 13.4.2011 - 9 C 2.10, Rdnr. 21; Thiemann, RdL 2017, 241, 242f.; Thiemann/Benz/Schumann, zfv 2015, 98, 103 f., https://doi.org/DOI 10.12902/zfv-0059-2015; Thomas, Flächenmanagement und Bodenordnung 2009, 56, 61 f.; Thiemann, RdL 2008, 257, 258-260; Glitz, RdL 2006, 29, 30. Anderer Ansicht Weiß, RdL 2017, 57-60.

57) Vgl. OVG Weimar, Urt. v. 17.1.2002 - 7 F 944/00, II.2 und II.3; Bundesregierung, Entwurf eines Gesetzes zur Änderung des Flurbereinigungsgesetzes 1994, BT-Drs.12/7909, S. 6 f.

58) Millenium Ecosystem Assessment, Ecosystems and Human Well-being: Synthesis, 2005; TEEB, The Economics of Ecosystems and Biodiversity, Ecological and Economic Foundations, 2011; Naturkapital Deutschland - TEEB DE, Der Wert der Natur für Wirtschaft und Gesellschaft - Eine Einführung, 2012; IPBES, Global assessment report on biodiversity and ecosystem services of the Intergovernmental Science-Policy Platform on Biodiversity and Ecosystem Services, 2019.

59) Ausführlich z.B. Schumacher/Schumacher in: Schumacher/Fischer-Hüftle, BNatSchG, 3. Aufl. 2021, \1 BNatSchG 47-55, 93-162.

60) IPBES, Summary for policymakers of the global assessment report on biodiversity and ecosystem services of the Intergovernmental Science-Policy Platform on Biodiversity and Ecosystem Services, 2019, S. 22f.; von Haaren/Albert, Ökosystemleistungen in ländlichen Räumen - Grundlage für menschliches Wohlergehen und nachhaltige wirtschaftliche Entwicklung, Naturkapital Deutschland TEEB DE, 2016, S. 30, 158-160; TEEB, The Economics of Ecosystems and Biodiversity, Ecological and Economic Foundations, 2011; Naturkapital Deutschland - TEEB DE, Der Wert der Natur für Wirtschaft und Gesellschaft, 2012, S. 24-30.

61) Von Haaren/Albert, Ökosystemleistungen in ländlichen Räumen, Naturkapital Deutschland TEEB DE, 2016, S. 77, 88-90.

62) Costanza u. a., Nature 1997, 253-260; von Haaren/Albert, Ökosystemleistungen in ländlichen Räumen, Naturkapital Deutschland TEEB DE, 2016, S. 50-57, 98-106; TEEB, The Economics of Ecosystems and Biodiversity, Ecological and Economic Foundations, 2011; Naturkapital Deutschland - TEEB DE, Der Wert der Natur für Wirtschaft und Gesellschaft, 2012, S. 30-36. 
Tabelle 1 Bewertung der Relevanz von Regulierungs- und Erhaltungsleistungen für die land- und forstwirtschaftlichen Versorgungsleistungen ${ }^{63}$

\begin{tabular}{|c|c|c|c|}
\hline $\begin{array}{l}\text { Regulierungs- und } \\
\text { Erhaltungsleistungen }\end{array}$ & Versorgungsleistungen & $\begin{array}{l}\text { bei landwirtschaftlichen } \\
\text { Flächen }\end{array}$ & $\begin{array}{l}\text { bei forstwirtschaftlichen } \\
\text { Flächen }\end{array}$ \\
\hline \multirow{5}{*}{$\begin{array}{l}\text { Erhaltung von physi- } \\
\text { kalischen, chemischen } \\
\text { und biologischen Be- } \\
\text { dingungen }\end{array}$} & $\begin{array}{l}\text { Erhaltung von Lebenszyklen, Habitaten und Gen- } \\
\text { pools (u.a. Bestäubung und Diasporenverbreitung, } \\
\text { Erhaltung von Aufzuchtpopulationen und -habitaten) }\end{array}$ & sehr hoch & hoch \\
\hline & $\begin{array}{l}\text { Schädlings- und Krankheitskontrolle (u.a. Förderung } \\
\text { der Pflanzengesundheit durch z.B. Mykorrhizapilze } \\
\text { und Reduktion von Schädlingen durch z.B. Gallwes- } \\
\text { pen und Vögel) }\end{array}$ & hoch & sehr hoch \\
\hline & $\begin{array}{l}\text { Bodenbildung, -aufbau und -zusammensetzung (u.a. } \\
\text { Humusbildung und Nährstofffreisetzung, Auflocke- } \\
\text { rung der Böden) }\end{array}$ & sehr hoch & sehr hoch \\
\hline & $\begin{array}{l}\text { Wasserqualität (u.a. Abbau und Fixierung von Schad- } \\
\text { stoffen, Regulierung des Säuregehalts) }\end{array}$ & $\begin{array}{l}\text { mittel (hoch in Tierhaltung } \\
\text { und Fischzucht) }\end{array}$ & hoch \\
\hline & $\begin{array}{l}\text { Luftqualität und Klimaregulierung (u.a. globaler und } \\
\text { lokaler Klimaverhältnisse) }\end{array}$ & hoch & hoch \\
\hline \multirow{2}{*}{$\begin{array}{l}\text { Regulierung von Stof- } \\
\text { fen (Bildung, Umwand- } \\
\text { lung, Bindung etc.) }\end{array}$} & $\begin{array}{l}\text { Regulierung durch Lebewesen (u.a. Photosynthese, } \\
\text { mikrobiologische Prozesse im Boden) }\end{array}$ & sehr hoch & sehr hoch \\
\hline & $\begin{array}{l}\text { Regulierung durch ökosystemare Prozesse (u.a. Was- } \\
\text { ser- und Nährstoffspeicherung in Böden) }\end{array}$ & hoch & hoch \\
\hline \multirow[t]{3}{*}{$\begin{array}{l}\text { Regulierung von } \\
\text { Massenbewegungen }\end{array}$} & $\begin{array}{l}\text { (Fest-)Massenbewegung (u.a. Reduzierung von } \\
\text { Erosion) }\end{array}$ & sehr hoch & mittel \\
\hline & $\begin{array}{l}\text { Wasserhaushalt und -abfluss (u.a. Menge und Zeit- } \\
\text { punkt von Niederschlägen und ihre Aufnahme und } \\
\text { Speicherung in Böden) }\end{array}$ & sehr hoch & sehr hoch \\
\hline & $\begin{array}{l}\text { Luft- und Gasmassenbewegung (u.a. Häufigkeit und } \\
\text { Intensität von Stürmen und von vom Wind beförder- } \\
\text { ten Austrocknungsprozessen) }\end{array}$ & hoch & sehr hoch \\
\hline
\end{tabular}

lokale Klima verändert wird. ${ }^{64}$ Die hieraus resultierenden ökonomischen Gesamtkosten übersteigen im Bereich der Landwirtschaft die volkswirtschaftliche Bruttowertschöpfung um ein Vielfaches. ${ }^{65}$

Wenn die Leistungen der Natur nicht nur dem Allgemeinwohl, sondern in erheblichem Umfang auch den wirtschaftlichen Interessen der privaten Eigentümer und Nutzer von Grundstücken dienen, dann lassen sich Maßnahmen zum Erhalt bzw. zur Wiederherstellung von Ökosystemen, natürlichen Prozessen sowie Habitaten und biologischer Vielfalt nicht per se als fremdnützig im Rahmen von Flurbereinigungsverfahren einstufen. ${ }^{66}$ Auch ein gemeinwohlbezogener Anlass schließt die Privatnützigkeit eines Flurbereinigungsverfahrens nicht aus. ${ }^{67}$ Vielmehr kommt es darauf an, ob die

63) Kategorisierung angelehnt an von Haaren/Albert, Ökosystemleistungen in ländlichen Räumen, Naturkapital Deutschland TEEB DE, 2016, S. 30

64) Siehe Abschnitt 1 und Hampicke, Kulturlandschaft - Äcker, Wiesen, Wälder und ihre Produkte, Berlin 2018, S. 77-112, 126128, 135-138; v.d. Decken u. a., Agrar-Report 2017 - Biologische Vielfalt in der Agrarlandschaft, BfN, 2017, S. 6-23, 36-41; von Haaren/Albert, Ökosystemleistungen in ländlichen Räumen, $\mathrm{Na}$ turkapital Deutschland TEEB DE, 2016, S. 92-97, $168 \mathrm{f}$.

65) Boston Consulting Group, Die Zukunft der deutschen Landwirtschaft nachhaltig sichern - Denkanstöße und Szenarien für ökologische, ökonomische und soziale Nachhaltigkeit, 2019, S. 16-23.

66) Binder, Flurbereinigungsplanung und Ökosystemschutz als Rechts- und Governance-Problem, Marburg 2019, S. 253-258; Thomas, RdL 2014, 293, 294; Wingerter in: Wingerter/Mayr, FlurbG, Standardkommentar, 10. Aufl., 2018, $\$ 1$ Rdnr. 7.

67) BVerwG, Beschl. v. 20.9.2019 - 9 B 50.18, Rdnr. 3; BVerwG, Beschl. v. 18.11.2014 - 9 B 30.14, Rdnr. 5. jeweilige Maßnahme im konkreten Flurbereinigungsgebiet gemäß $\$ \$ 4$, 39 FlurbG überwiegend die gemeinschaftlichen Interessen der Teilnehmer fördert oder v. a. die Allgemeinheit davon profitiert. ${ }^{68} \mathrm{Im}$ Folgenden soll dies beispielhaft an ausgewählten Themenfeldern erörtert werden.

\subsubsection{Maßnahmen zur Klimaanpassung}

Die klimatischen Veränderungen aufgrund steigender Treibhausgase und Durchschnittstemperaturen werden auch in Deutschland zunehmen und können je nach Region und Jahreszeit u. a. zu geringeren Niederschlägen und längeren Dürreperioden, aber auch zu mehr Wetterextremereignissen führen. ${ }^{69}$ In Anbetracht des langfristigen Zeithorizonts der Flurbereinigung stehen Maßnahmen, welche

68) Vgl. BVerwG, Beschl. v. 20.9. 2019 - 9 B 50.18, Rdnr. 3-5.

69) Renner u.a., Klimawirkungs- und Risikoanalyse 2021 für Deutschland - Teilbericht 2: Risiken und Anpassung im Cluster Land, 2021; Fritsch u. a., Klimawirkungs- und Risikoanalyse 2021 für Deutschland - Teilbericht 3: Risiken und Anpassung im Cluster Wasser, 2021; DKM u. a., Was wir heute übers Klima wissen, Berlin 2020, S. 13-19; EEA, Climate change adaptation in the agriculture sector in Europe, EEA Report No 04/2019, S. 42-69; Brasseur/Jacob/Schuck-Zöller, Klimawandel in Deutschland - Entwicklung, Folgen, Risiken und Perspektiven, 2017; Steininger/Wurbs, Bundesweite Gefährdung der Böden durch Winderosion und Bewertung der Veränderung infolge des Wandels klimatischer Steuergrößen als Grundlage zur Weiterentwicklung der Vorsorge und Gefahrenabwehr im Bodenschutzrecht, 2017, S. 80-89; Heißenhuber/Haber/Krämer, 30 Jahre SRU - Sondergutachten „Umweltprobleme der Landwirtschaft“ eine Bilanz, 2015, S. 147-155. 
die zu erwartenden Folgen des Klimawandels bei Flächen im Flurbereinigungsgebiet abmildern, im objektiven Interesse der Grundstückseigentümer und Erbbauberechtigten, da sie dem langfristigen Erhalt der land- und forstwirtschaftlichen Bewirtschaftbarkeit von Flächen dienen. ${ }^{70}$ Dies ist auch anzunehmen, wenn dadurch die gegenwärtige Nutzung von Flächen eingeschränkt oder verändert wird, solange damit eine langfristige land- und forstwirtschaftliche Nutzung der Flächen gesichert wird. Hierin unterscheidet sich die Klimaanpassung von Maßnahmen, die allein dem Klimaschutz dienen, da bei letzteren ein positiver Effekt für das Flurbereinigungsgebiet aufgrund der globalen Zusammenhänge marginal ist. Als privatnützig im Sinne der Flurbereinigung u.a. folgende Maßnahmen zur Anpassung an den Klimawandel anzusehen ${ }^{71}$ :

- die Wiedereinräumung von Landschaftselementen (z.B. Hecken, Baumreihen, im Rahmen von Agroforstsystemen) zum Schutz vor Wind- und Wassererosion sowie zur Verringerung der Austrocknung von Böden durch Wind und Sonne,

- die Erhöhung bzw. Wiederherstellung der Diversität bei landwirtschaftlichen Kulturpflanzen sowie forstlichen Baumarten (einschließlich Altersdiversität), die Resilienz gegenüber Dürren und Extremwetterereignissen zu erhöhen,

- der Rückbau von Entwässerungsgräben und Drainagen, um den Grundwasserspiegel wieder anzuheben und das Regenwasser länger auf und in den Böden $z u$ halten,

- der Schutz und die Wiederanlage von Kleingewässern (z.B. Teiche), Sümpfen und Mooren, um das lokale Klima zu regulieren,

- die Umwandlung von erosionsgefährdeten Ackerflächen in Dauerkulturen (z.B. Dauergrünland, Wald, Agroforstsysteme, Streuobstwiesen) sowie

- die Erhöhung des Humusgehalts und die Verbesserung der biologischen Vielfalt in Böden, um deren Fähigkeiten zur Wasserinfiltration und -speicherung zu erhöhen. 72

Nach der Ackerbaustrategie des BMEL sollen daher Flurbereinigungsverfahren zur Klimaanpassung stärker auf Bodenschutz und Erosionsminderung ausgerichtet sowie mit ihrer Hilfe Bewässerungsinfrastrukturen aufgebaut werden. ${ }^{73}$ Während Bodenschutz und Erosionsminderung die Ökosystemleistungen im Interesse der Grundstückseigentümer und Bewirtschafter befördern, können Bewässerungsmaßnahmen mit einer Verschlechterung von Ökosystemen und ihrer Leistungen einhergehen. Bevor derartige Bewässerungsanlagen in einem Wege- und Gewässerplan als gemeinschaftliche Anlagen im Sinne von $₫ 39$ FlurbG festgesetzt werden können, müssen sie gemäß $\$ 37$ Abs. 2 FlurbG mit den konkurrierenden Belangen der Wasserwirtschaft einschließlich der Wasserversorgung sowie dem Umwelt- und Naturschutz abgewogen werden.

\subsubsection{Maßnahmen zum Erhalt oder}

zur Wiederherstellung der biologischen Vielfalt

Wildlebende Tier- und Pflanzenarten bilden die Ökosysteme und beeinflussen in vielfältiger Weise die Lebensbedingungen auf der Erde (z.B. Produktion von Sauerstoff und Biomasse, Regulierung des Klimas, Abbau von Restund Schadstoffen). ${ }^{74}$ Als Lebensgrundlage für alle biologischen Wesen hat der Rückgang der biologischen Vielfalt und Populationsgrößen auch negative Auswirkungen auf die menschlichen Lebensgrundlagen, ${ }^{75}$ deren Schutz vor anthropogenen Beeinträchtigungen das Bundesverfassungsgericht eine herausragende Bedeutung für die Sicherung der individuellen Grundfreiheiten (u.a. Leben und körperliche Unversehrtheit, Eigentum- und Berufsfreiheit) zumisst. ${ }^{76}$

Eine Vielzahl der in der Tabelle genannten Regulierungs- und Erhaltungsleistungen mit Relevanz für die land- und forstwirtschaftliche Bodennutzung beruht auf dem Wirken und Interagieren von wildlebenden Tier- und Pflanzenarten. Hervorzuheben sind u. a.:

- Wildlebende Insekten erbringen umfangreiche Bestäubungsleistungen für land- und forstwirtschaftliche Kulturen. ${ }^{77}$

- Nützlinge wie Vögel, konkurrierende Insekten oder attraktive Wildpflanzen reduzieren den Schädlingsdruck auf Kulturpflanzen. ${ }^{78}$

- Verschiedenste Bodenlebewesen sichern die Bodenfruchtbarkeit und sorgen u. a. für den Aufbau von $\mathrm{Hu}-$ mus sowie gute Infiltrations- und Speichereigenschaften von Böden. ${ }^{79}$

70) Vgl. Topagrar 2017, 20-27; Möckel, UPR 2012, 247, 253

71) Vgl. BVerwG, Beschl. v. 20.9.2019 - 9 B 50.18, Rdnr. 5; OVG Bautzen, Urt. v. 29.1.2018 - 7 C 22/16.F, Rdnr. 26-28; VG Saarlouis, Urt. v. 24.11.1988 - 2 K 27/87, NuR 1990, 284 , 285; Binder, Flurbereinigungsplanung und Ökosystemschutz als Rechts- und Governance-Problem, Marburg 2019, S. 253-257; Thiemann, RdL 2017, 241, 242-244; Thomas, RdL 2014, 293, 295; Dippold, NuR 1990, 58, 61

72) Vgl. Zukunftskommission Landwirtschaft, Zukunft Landwirtschaft. Eine gesamtgesellschaftliche Aufgabe, 2021, S. $97 \mathrm{ff}$; BMEL, Ackerbaustrategie 2035 - Perspektiven für einen produktiven und vielfältigen Pflanzenbau, 2021, S. 38f.; EEA, Climate change adaptation in the agriculture sector in Europe, EEA Report No 04/2019, S. 74f.; Schwarzer, Putting Carbon back where it belongs - the potential of carbon sequestration in the soil, 2019; von Haaren/Albert, Ökosystemleistungen in ländlichen Räumen, Naturkapital Deutschland TEEB DE, 2016, S. 116-119; Blume/ Horn/Thiele-Bruhn, Handbuch des Bodenschutzes, Weinheim 2011, S. 575-577; Hartmann u. a., Vergleichende Untersuchungen der Infiltrationseigenschaften von konventionell und ökologisch bewirtschafteten Böden, Eine Fallstudie aus dem Main-Tauber Kreis, Baden-Württemberg, 2009; Eastaugh u.a., Forest Agencies' Early Adaptations to Climate Change, Wien 2009, S. 30-33; Schnug/Haneklaus, Landbauforschung Völkenrode 2002, 197-203.

73) BMEL, Ackerbaustrategie 2035 - Perspektiven für einen produktiven und vielfältigen Pflanzenbau, 2021, S. 20, 39, 55.

74) Vgl. IPCC/IPBES, Scientific Outcome of the IPBES-IPCC cosponsored workshop on biodiversity and climate change, 2021; IPBES, Global assessment report on biodiversity and ecosystem services of the Intergovernmental Science-Policy Platform on Biodiversity and Ecosystem Services, 2019. Anerkennend Zukunftskommission Landwirtschaft, Zukunft Landwirtschaft. Eine gesamtgesellschaftliche Aufgabe, 2021; BMEL, Ackerbaustrategie 2035 - Perspektiven für einen produktiven und vielfältigen Pflanzenbau, 2021, S. 35.

75) Vgl. Cardinale u.a., Nature 2012, 59-65, https://doi.org/10. 1038/nature11148.

76) BVerfG, Beschl. v. 23.3.2021 - 1 BvR 2656/18 u.a.

77) Leopoldina/acatech/Akademienunion, Biodiversität und Management von Agrarlandschaften, 2020, S. 18f.; IPBES, Assessment Report on Pollinators, Pollination and Food Production, 2017, Kap. 1 und 4; von Haaren/Albert, Ökosystemleistungen in ländlichen Räumen, Naturkapital Deutschland TEEB DE, 2016, S. 104-107; Knauer, Ökologie und Landwirtschaft: Situation, Konflikte, Lösungen, Stuttgart 1993, S. 143-145.

78) Barnes u.a., Science Advances 2020, https://doi.org/10.1126/sciadv. abb6603; Leopoldina/acatech/Akademienunion, Biodiversität und Management von Agrarlandschaften, 2020, S. 19; von Haaren/Albert, Okosystemleistungen in ländlichen Räumen, Naturkapital Deutschland TEEB DE, Hannover, Leipzig 2016, S. 100-104; Altieri/Ponti/Nicholls, Soil fertility, biodiversity and pest management, in: Gurr/Wratten/Snyder/Read (Hrsg.): Biodiversity and insect pests: key issues for sustainable management, Hoboken, New Jersey 2012, S. 72-84; Knauer, Ökologie und Landwirtschaft: Situation, Konflikte, Lösungen, Stuttgart 1993, S. 107, 179 f.

79) BMEL, Ackerbaustrategie 2035 - Perspektiven für einen produktiven und vielfältigen Pflanzenbau, 2021, S. 19; Schwarzer Putting Carbon back where it belongs - the potential of carbon sequestration in the soil, 2019; Hartmann u. a., Vergleichende Untersuchungen der Infiltrationseigenschaften von konventionell und ökologisch bewirtschafteten Böden, Eine Fallstudie aus dem Main-Tauber Kreis, Baden-Württemberg, Braunschweig 2009; Blume/Horn/Thiele-Bruhn, Handbuch des Bodenschutzes, Weinheim 2011, S. 110-112. 
- Bodenmikroorganismen wie Mykorrhizapilze befördern die Gesundheit von Kulturpflanzen und sorgen für eine bessere Wasserversorgung der Pflanzen in Trockenzeiten. ${ }^{80}$

Flurbereinigungsmaßnahmen, welche die biologische Vielfalt im Gebiet erhöhen, indem sie in Agrarlandschaften für wildlebende Arten Habitate erhalten oder wiederherstellen (z.B. durch die Extensivierung von Nutzungsbereichen oder die Schaffung von Landschaftselementen wie Hecken, Kleingewässer, Blühstreifen und Bauminseln ${ }^{81}$ und in Wäldern günstige Lebens- und Nahrungsbedingungen schaffen ${ }^{82}$, sind daher regelmäßig als privatnützig im Sinne von $\$ 4$ FlurbG und als gemeinschaftliche Anlagen im Sinne von $\$ 39$ FlurbG einzustufen, da sie eine dauerhaft umweltgerechte Landbewirtschaftung ermöglichen. Diese positiven lokalen Wirkungen überwiegen dabei regelmäßig die damit einhergehenden Vorteile für die Allgemeinheit. ${ }^{83}$

Maßnahmen zur Wiederherstellung von Habitaten und der biologischen Vielfalt erhöhen regelmäßig zugleich die Attraktivität einer Landschaft als Lebensraum für die Menschen. ${ }^{84}$ Nicht nur eine höhere touristische Attraktivität mit neuen Einkommensperspektiven, sondern auch die Attraktivität als Wohnort wirkt der in vielen ländlichen Gebieten vorherrschenden Abwanderung entgegen, was sich unmittelbar auf die monetären Marktwerte der Grundstücke im Gebiet positiv auswirkt und damit geldwerte Vorteile für die Teilnehmer schafft. ${ }^{85}$

\subsubsection{Maßnahmen zur Umsetzung} umweltrechtlicher Anforderungen

Viele Flächen im ländlichen Raum unterliegen naturschutz- oder umweltrechtlichen Anforderungen, welche als Inhalts- und Schrankenbestimmungen im Sinne von Art. 14 Abs. 1 GG die Eigentümerbefugnisse einschränken. Sie sind Ausdruck der nach Art. 14 Abs. 2 GG gebotenen Sozialpflichtigkeit von Eigentum, welche bei Grundeigentum aufgrund der Unvermehrbarkeit sowie Situationsgebundenheit von Grund und Boden besonders hoch ist (siehe 3.1).

Umweltrechtliche Inhaltsbestimmungen des Grundeigentums sind neben den allgemeinen Anforderungen an die gute fachliche Praxis der land-, forst- und fischereiwirtschaftlichen Bodennutzung (z.B. im Dünge- und Pflanzenschutzrecht) ${ }^{86}$ auch die Verpflichtungen aus der Eingriffsregelung in $\$ \$ 14-17$ BNatSchG. Soweit im Rahmen der Flurbereinigung Landschaftselemente entfernt werden, der Grundwasserspiegel abgesenkt wird oder z. B. Dauergrünland in Acker umgewandelt wird, sind dies Eingriffe

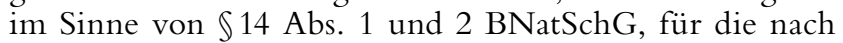
$\int 15$ Abs. 2 BNatSchG ein Ausgleich oder Ersatz zu schaffen ist. ${ }^{87}$ Die Kompensationsmaßnahmen sind daher gemeinschaftliche Anlagen der Teilnehmergemeinschaft. ${ }^{88}$

Den Inhalt des Grundeigentums bestimmen weiterhin flächenspezifische Vorgaben für gesetzlich geschützte Biotope ( $(30$ BNatSchG und Landesrecht) sowie für Gewässerrandstreifen und Überschwemmungsgebiete (\$S $38,38 \mathrm{a}$, 78a WHG und Landesrecht) und insbesondere Festsetzungen im Rahmen von Schutzgebietsausweisungen ( $\$ \int 23-$ 29, 32-34 BNatSchG und Landesrecht, \$\$ 51, 52 WHG). Etliche der Inhaltsbestimmungen erfolgen in Umsetzung internationaler Abkommen zum Schutz von Ökosystemen und wildlebenden Tier- und Pflanzenarten ${ }^{89}$ sowie europäischer Rechtsakte (insbesondere Flora-Fauna-HabitatRichtlinie 92/43/EWG, Vogelschutz-Richtlinie 2009/147/ EG, Wasser-Rahmen-Richtlinie 2000/60/EG, Hochwasser-Management-Richtlinie 2007/60/EG). Sowohl die FFH-Richtlinie als auch die Wasser-Rahmen-Richtlinie verpflichten Deutschland, gute ökologische Zustände bei einer Vielzahl besonders schutzwürdiger Lebensraumtypen und Arten sowie grundsätzlich bei allen Oberflächengewässern zu erreichen. Gute ökologische Zustände gewähr- leisten, dass die betreffenden Regulierungs- und Erhaltungsleistungen dauerhaft generiert werden.

Soweit Flurbereinigungsverfahren dazu dienen, allgemeine oder flächenspezifische Vorgaben des Rechts an Landnutzungen im Flurbereinigungsgebiet besser umzusetzen und bestehende Konflikte zu beseitigen, liegt dies im objektiven Interesse der Teilnehmer an einer rechtskonformen Landgestaltung und Bodennutzung. ${ }^{90}$ Auch der damit einhergehende dauerhafte Erhalt bzw. die Wiederherstellung ökologischer Regulierungs- und Erhaltungsleistungen dient den Interessen der Grundstückseigentümer und der land- und forstwirtschaftlichen Bewirtschafter. Sofern in Zukunft rechtliche Anforderungen an eine klimaschützende land- und forstwirtschaftliche Bodennutzung normiert werden, ist deren Erfüllung ebenfalls im Interesse der Teilnehmer. Die persönliche Ablehnung der rechtlichen Vorgaben durch einzelne oder selbst durch alle Teilnehmer ist dabei unbeachtlich, da rechtswidrige Landnutzungen und Zustände nicht im objektiven Interesse sind. Maßnahmen zur Herstellung rechtskonformer Zustände sind daher auch gemeinschaftliche Anlagen im Sinne von $\$ 39$ FlurbG, selbst wenn die rechtlichen Vorgaben auch

80) Finckh u.a., Pflanzengesundheit richtig managen, Ökologie \& Landbau 2021, 41 ff.; Van Bruggen u. a., Sci. Total Environ. 2019, 927-937, https://doi.org/https://doi.org/10.1016/j.scitotenv. 2019.02.091; Altieri/Ponti/Nicholls, Soil fertility, biodiversity and pest management, in: Gurr/Wratten/Snyder/Read (Hrsg.): Biodiversity and insect pests: key issues for sustainable management, Hoboken, New Jersey 2012, S. 72-84; Blume/Horn/Thiele-Bruhn, Handbuch des Bodenschutzes, Weinheim 2011, S. 112.

81) Vgl. Zukunftskommission Landwirtschaft, Zukunft Landwirtschaft. Eine gesamtgesellschaftliche Aufgabe, 2021, S. $100 \mathrm{ff}$.; BMEL, Ackerbaustrategie 2035 - Perspektiven für einen produktiven und vielfältigen Pflanzenbau, Berlin, S. 35 f.; Beiträge in: Kollmann/Kirmer/Tischew u.a., Renaturierungsökologie, 2019; Zerbe, Renaturierung von Ökosystemen im Spannungsfeld von Mensch und Umwelt, 2019. Vgl. auch die Ergebnisse der Metastudie Martin u.a., Ecology Letters 2019, 1083-1094, https://doi.org/https://doi.org/10.1111/ele.13265.

82) Vgl. Zerbe, Renaturierung von Ökosystemen im Spannungsfeld von Mensch und Umwelt, 2019, $107 \mathrm{ff}$; Hölzel, in: Kollmann/ Kirmer/Tischew u.a., Renaturierungsökologie, 2019, $101 \mathrm{ff}$.

83) Vgl. OVG Koblenz, Urt. v. 15.1.2014 - 9 C 10644/13, Rdnr. 2732, RdL 2015, 65-68; Bundesregierung, Entwurf eines Gesetzes zur Änderung des Flurbereinigungsgesetzes 1994, BTDrs.12/7909, S. 8; VG Saarlouis, Urt. v. 24.11. 1988 - 2 K 27/87, NuR 1990, 284, 285.

84) Vgl. Heißenhuber/Haber/Krämer, 30 Jahre SRU - Sondergutachten ,Umweltprobleme der Landwirtschaft - eine Bilanz, 2015, S. 179.

85) Siehe z.B. die Entwicklung der Bevölkerungszahl und der Immobilienpreise im Biosphärenreservat Schorfheide-Chorin (vgl. Lehmann, Morgenpost 2018, article214605075; Amt für Statistik Berlin-Brandenburg, Bevölkerung im Land Brandenburg von 1991 bis 2019 nach Kreisfreien Städten, Landkreisen und Gemeinden, Tabelle 7: Schorfheide 12060198, 2020).

86) Die Vorgaben in $₫ 17$ Abs. 2 BBodSchG und $₫ 5$ Abs. 2 BNatSchG sind hingegen unverbindlich (vgl. $\$ 17$ Abs. 1 BBodSchG; BVerwG, Urt. v. 1.9.2016-4 C 4.15).

87) Vgl. BVerwG, Urt. v. 13.4.1983 - C 76.80, Rdnr. 94; BVerwG, Beschl. v. 29.11.1985 - 4 B 213.85, NuR 1986, 251; BVerwG, Beschl. v. 16.2.1992 - 4 B 38.92, NuR 1992, 328, 328f.; BVerwG, Beschl. v. 4.6.2003 - 4 BN 27.03; Möckel, NuR 2012, 225, 225-229.

88) Binder, Flurbereinigungsplanung und Ökosystemschutz als Rechts- und Governance-Problem, Marburg 2019, S. 338-341; Möckel, UPR 2012, 247, 254.

89) UN, Convention on Biological Diversity 1992; CMS, Convention on the Conservation of Migratory Species of Wild Animals, 1979; COE, Convention on the Conservation of European Wildlife and Natural Habitats 1979; Ramsar Convention on Wetlands 1971.

90) Vgl. BVerwG, Beschl. v. 18.11.2014 - 9 B 30.14, Rdnr. 5; BVerwG, Urt. v. 13. 4. 2011 - 9 C 2.10, Rdnr. 21. 
oder gar überwiegend überregionalen Allgemeinwohlinteressen dienen.

\section{Fazit}

Flurbereinigungsverfahren sind privatnützig, wenn sie dem objektiven Interesse der Gemeinschaft der Grundstückseigentümer im betreffenden Gebiet dienen. Das objektive Interesse umfasst nicht nur ökonomische, sondern auch soziale und ökologische Interessen. Da Flurbereinigungsverfahren für längere Zeiträume ein Gebiet rechtlich neu ordnen und landschaftlich umgestalten sollen, stehen langfristige Interessen im Vordergrund. Im objektiven Interesse ist weiterhin, dass nicht nur zweckmäßige, sondern auch rechtskonforme Zustände im Gebiet geschaffen werden.

Das Konzept der Ökosystemleistungen verdeutlicht, dass die Land- und Forstwirtschaft mit ihren Versorgungsleistungen wesentlich auf die von Okosystemen und wildlebenden Arten bereitgestellten Regulierungs- und Erhaltungsleistungen angewiesen ist und auch die sogenannten kulturellen Ôkosystemleistungen ökonomische und soziale Vorteile für die Teilnehmer eines Flurbereinigungsgebietes erbringen. Der Erhalt und die Wiederherstellung von Ökosystemleistungen sichern und befördern die land- und forstwirtschaftliche Bewirtschaftung von Grundstücken im Sinne von $\$ 1$ FlurbG. Es verbessert den Zustand und auch den ökonomischen Wert von Grund und Boden, wenn z. B. Bienenpopulationen (die als Bestäuber dienen) und Vögel (die zur Dezimierung von Schadinsekten beitragen) gute Lebensbedingungen haben, Landschaftsstrukturen der Erosion von Böden vorbeugen, ein ausgeglichener Bodenund Landschaftswasserhaushalt die Folgen von Dürren minimiert oder der Waldumbau klimastabile Wälder befördert. Zudem steigt der ökonomische Grundstückswert, wenn sich im Gebiet die Wohn- und Erholungsqualität erhöht. Insgesamt schärft das Konzept der Ökosystemleistungen den Blick für die wertbildenden ökologischen Faktoren von Grundeigentum im Sinne von Art. 14 GG. Die Erreichung naturschutzrechtlicher Ziele - wozu der Erhalt und die Wiederherstellung der Leistungs- und Funktionsfähigkeit des Naturhaushaltes sowie der biologischen Vielfalt und des Erholungswerts von Natur und Landschaft (vgl. \$1 Abs. 1 BNatSchG) gehören - ist daher auch im objektiven Interesse der Eigentümer und Nutzer von Grundstücken.

Ökosystemleistungen sind auch von wesentlicher Bedeutung für eine integrierte Landentwicklung im Sinne von $\$ 1$ FlurbG. Der Erhalt und die Wiederherstellung von Ökosystemen und ihrer Leistungen dienen zwar auch dem Allgemeinwohl, regelmäßig überwiegen aber die lokalen Vorteile für die beteiligten Grundstückseigentümer. Damit können privatnützige Flurbereinigungsverfahren auch für Maßnahmen eingesetzt werden, welche im Flurbereinigungsgebiet die Anpassung an den Klimawandel befördern, die biologische Vielfalt erhalten bzw. wiederherstellen oder der Umsetzung umweltrechtlicher Anforderungen und der Schaffung rechtskonformer Zustände dienen. Derartige Maßnahmen sind keine öffentlichen Anlagen nach $\$ 40$ FlurbG, sondern gemeinschaftliche Anlage im Sinne von $\$ 39$ FlurbG, für die die Teilnehmer entsprechend Land und Finanzmittel bereitstellen müssen.

Der Einsatz der Flurbereinigung als Instrument zur Schaffung klimaangepasster, nachhaltiger Landschaften ließe sich vom Bundesgesetzgeber in der Praxis befördern, wenn in $\iint 1,4$ und 39 FlurbG der Erhalt und die Wiederherstellung von Ökosystemen und ihrer Leistungen ausdrücklich als Ziele der Flurbereinigung und Gegenstand privatnütziger Flurbereinigungsverfahren sowie gemeinschaftlicher Anlagen erwähnt würden, um mit diesen redaktionellen Klarstellungen bestehende Rechtsunsicherheiten bei der Auslegung der gegenwärtigen Regelungen zu beseitigen. Oppermann/Ostermann/Blew et al. plädieren sogar dafür, das FlurbG durch ein neu ausgerichtetes
Bodenordnungsgesetz zu ersetzen. ${ }^{91} \mathrm{Um}$ bei überregionalen Naturschutzprojekten, die regelmäßig im überwiegenden öffentlichen Interesse stehen (z.B. die Arrondierung größerer Schutzgebiete oder überregionaler Biotopverbindungen), die Möglichkeiten der fremdnützigen, enteignenden Unternehmensflurbereinigung effektiv und zum Wohle der Allgemeinheit nutzen zu können, müssten der Bund oder die Länder in ihren Naturschutzgesetzen für diese Ziele Planfeststellungsverfahren mit enteignungsrechtlicher Vorwirkung vorsehen.

In welchem Umfang das Flurbereinigungsrecht mit der Föderalismusreform im Jahr $2006^{92}$ in den Kompetenzbereich der Länder zurückgefallen ist, wurde bisher nicht höchstrichterlich geklärt. In der Literatur werden hierzu unterschiedliche Ansichten vertreten ${ }^{93}$, da die Flurbereinigung nur beim Kompetenztitel „Förderung der land- und forstwirtschaftlichen Erzeugung“, nicht aber beim „Bodenrecht", auf das sich das Flurbereinigungsgesetz ebenfalls stützt, herausgenommen wurde (vgl. Art. 74 Abs. 1 Nr. 17 und 18 GG). Daraus wird teilweise abgeleitet, dass die Zuständigkeit des Bundes für das materielle Recht der Flurbereinigungsgesetzgebung als ländliches Bodenrecht unverändert erhalten geblieben ist und auch kein Redaktionsversehen des Gesetzgebers vorliegt. ${ }^{94}$ Aber selbst wenn der Bund seine Gesetzgebungskompetenz vollständig abgegeben haben sollte, darf er im Rahmen des Art. 125a GG die Vorschriften seines Gesetzes weiterhin ändern, solange damit keine grundlegende Umgestaltung des Flurbereinigungsrechts verbunden ist. ${ }^{95}$ Letzteres ist bei redaktionellen Klarstellungen nicht der Fall, da diese nicht den normativen Inhalt ändern, sondern lediglich in der Praxis die Rechtssicherheit bei der Normanwendung erhöhen. Neben dem Bund können seit 2006 auch die Länder tätig werden und landesrechtlich das Flurbereinigungsrecht weiterentwickeln, wie es z.B. in Baden-Württemberg und Niedersachsen hinsichtlich veränderter Zielsetzungen geschehen ist. ${ }^{96}$

Open Access. Dieser Artikel wird unter der Creative Commons Namensnennung 4.0 International Lizenz veröffentlicht, welche die Nutzung, Vervielfältigung, Bearbeitung, Verbreitung und Wiedergabe in jeglichem Medium und Format erlaubt, sofern Sie den/die ursprünglichen Autor(en) und die Quelle ordnungsgemäß nennen, einen Link zur Creative Commons Lizenz beifügen und angeben, ob Änderungen vorgenommen wurden.

Die in diesem Artikel enthaltenen Bilder und sonstiges Drittmaterial unterliegen ebenfalls der genannten Creative Commons Lizenz, sofern sich aus der Abbildungslegende nichts anderes ergibt. Sofern das betreffende Material nicht unter der genannten Creative Commons Lizenz steht und die betreffende Handlung nicht nach gesetzlichen Vorschriften erlaubt ist, ist für die oben aufgeführten Weiterverwendungen des Materials die Einwilligung des jeweiligen Rechteinhabers einzuholen

Weitere Details zur Lizenz entnehmen Sie bitte der Lizenzinformation auf http://creativecommons.org/licenses/by/4.0/deed.de.

Open Access funding enabled and organized by Projekt DEAL.

91) Oppermann u. a., Flurbereinigung und Naturschutz, 2003, S. $23 \mathrm{f}$

92) Deutscher Bundestag, Gesetzentwurf der Fraktionen der CDU/ CSU und SPD: Entwurf eines Gesetzes zur Änderung des Grundgesetzes, BT-Drs. 16/813, S. 12-13, 2006.

93) Siehe Überblick bei Hönes, NuR 2014, 153, 155.

94) Weiß, RdL 2006, 309-314; Weiß, Zur Entwicklung des Flurbereinigungsgesetzes der Bundesrepublik Deutschland in den vergangenen 6 Jahrzehnten, Butjadingen-Stollham 2009, S. 60-64.

95) Binder, Flurbereinigungsplanung und Ökosystemschutz als Rechts- und Governance-Problem, Marburg 2019, S. 189192; Jarass/Pieroth, GG-Kommentar, 16. Aufl., 2020, Art. 125a Rdnr. 8; Maunz/Dürig, GG - Grundgesetz Kommentar, 92. Lfg., 2020, Art. 125a Rdnr. 27.

96) Vgl. Thomas, NuR 2014, 686, 691. 